\title{
High-performance position-sensitive detector based on the lateral photoelectrical effect of two-dimensional materials
}

\author{
Chang $\mathrm{Hu}^{1}$, Xianjie Wang ${ }^{1}$ and Bo Song ${ }^{2}$
}

\begin{abstract}
Two-dimensional (2D) materials such as graphene and transition-metal chalcogenides have been extensively studied because of their superior electronic and optical properties. Recently, 2D materials have shown great practical application in position-sensitive detectors (PSDs), originating from the lateral photoelectrical effect of the materials or junctions. The high position sensitivity and ultrafast photoresponse of PSDs based on 2D materials, especially compatibility with Si technology, may enable diverse optoelectronic applications. In this review, recent studies of PSDs based on 2D materials are summarized, providing a promising route for high-performance PSDs.
\end{abstract}

\section{Introduction}

Since Schottky and Wallmark discovered and promoted the lateral photovoltaic effect (LPE) in $p-n$ junctions ${ }^{1}$, intensive research has been conducted on the LPE in diverse applications, including space exploration, environmental monitoring, and optical engineering. In the LPE, when one side of a $p-n$ junction is illuminated by a light spot, the optically excited electron-hole pairs will be separated up and down by the built-in electric field. Notably, the formation mechanism of the built-in field in position-sensitive detectors (PSDs) with different architectures is different. As shown in Fig. 1a, the PSD based on a $p-n$ junction uses the built-in field formed at the interface due to different band structures to separate the photogenerated carriers. The built-in field in the PSD based on the insulator-coated substrate (such as a graphene transistor on a $\mathrm{SiO}_{2} / \mathrm{Si}$ substrate) is formed by band energy bending induced by the localized interface states, and the photogenerated carriers accumulate at the $\mathrm{SiO}_{2} /$ $\mathrm{Si}$ interface and then diffuse laterally, as shown in Fig. 1b.

\footnotetext{
Correspondence: Bo Song (songbo@hit.edu.cn)

${ }^{1}$ Department of Physics, Harbin Institute of Technology, 150001 Harbin, China ${ }^{2}$ National Key Laboratory of Science and Technology on Advanced Composites in Special Environments, Harbin Institute of Technology, 150001 Harbin, China These authors contribued equally: Chang Hu, Xianjie Wang
}

The mechanism of carrier lateral diffusion can be explained by the model shown in Fig. 1c. The photogenerated electrons enter the $n$-type semiconductor, while the photogenerated holes enter the $p$-type semiconductor. Then, these generated carriers diffuse laterally from the light spot to balance the electric field along the layer ${ }^{2-6}$. In general, the relationship between the total number of photogenerated electrons $n_{0}$ and that of photogenerated electrons transmitted into $n$-type semiconductor $N_{0}$ can be described as follows:

$$
N_{0}=n_{0}\left[1-P^{\tau p / n_{0}}\right]
$$

where $P$ is the probability of photogenerated electrons entering the $n$-type semiconductor, $p$ is the laser power, and $\tau$ is a time-dependent coefficient.

The electron diffusion equation at position $r$ is

$$
D_{\mathrm{n}} \frac{d^{2} N(r)}{\mathrm{dr}^{2}}=\frac{N(r)}{\tau_{\mathrm{n}}}
$$

\section{(c) The Author(s) 2020}

(c) (i) Open Access This article is licensed under a Creative Commons Attribution 4.0 International License, which permits use, sharing, adaptation, distribution and reproduction (c) in any medium or format, as long as you give appropriate credit to the original author(s) and the source, provide a link to the Creative Commons license, and indicate if changes were made. The images or other third party material in this article are included in the article's Creative Commons license, unless indicated otherwise in a credit line to the material. If material is not included in the article's Creative Commons license and your intended use is not permitted by statutory regulation or exceeds the permitted use, you will need to obtain permission directly from the copyright holder. To view a copy of this license, visit http://creativecommons.org/licenses/by/4.0/. 
From these equations, the electron density $N(\mathrm{r})$ can be derived as follows:

$$
N(\mathrm{r})=N_{0} \exp \left(-\frac{|x-r|}{\lambda_{\mathrm{n}}}\right)
$$

where $\lambda_{\mathrm{n}}=\sqrt{D_{\mathrm{n}} \tau_{\mathrm{n}}}$ is the diffusion length, $D_{\mathrm{n}}=\frac{k_{\mathrm{B}} T}{\mathrm{e}^{2} \rho N_{\mathrm{F} 0}}$, and $\rho$ are the diffusion constant and resistivity of the $n$-type semiconductor, respectively; $\tau_{\mathrm{n}}$ is the electron diffusion lifetime, $N_{\mathrm{F} 0}=\frac{8 \pi}{3}\left(\frac{2 m_{e} E_{\mathrm{F}}}{h^{2}}\right)^{\frac{3}{2}}$ is the electron density below the Fermi level $\left(E_{\mathrm{F} 0}\right)$, and $x$ is the position of the laser point.

The Fermi level of an $n$-type semiconductor after laser irradiation at position $\mathrm{r}$ can be expressed as follows:

$$
E_{\mathrm{Fn}}(r)=E_{\mathrm{F} 0}+\frac{k_{\mathrm{B}} T}{n_{\mathrm{T}}} N(r)
$$

where the electron density in the n-type semiconductor conduction band is

$$
n_{\mathrm{T}}=\frac{2\left(2 \pi m_{\mathrm{e}} k_{\mathrm{B}} T\right)^{\frac{3}{2}}}{h^{3}} \exp \left(-\frac{E_{\mathrm{C}}-E_{\mathrm{F} 0}}{k_{\mathrm{B}} T}\right)
$$

Using the above formulas, a theoretical formula for the relationship between the LPE and laser irradiation position can be obtained as follows:

$$
\mathrm{LPE}=\frac{E_{\mathrm{Fn}}(\mathrm{L})-E_{\mathrm{Fn}}(-L)}{e}=\mathrm{Kn}_{0}\left[\exp \left(-\frac{|L-x|}{\lambda_{\mathrm{n}}}\right)-\exp \left(-\frac{|L+x|}{\lambda_{\mathrm{n}}}\right)\right]
$$

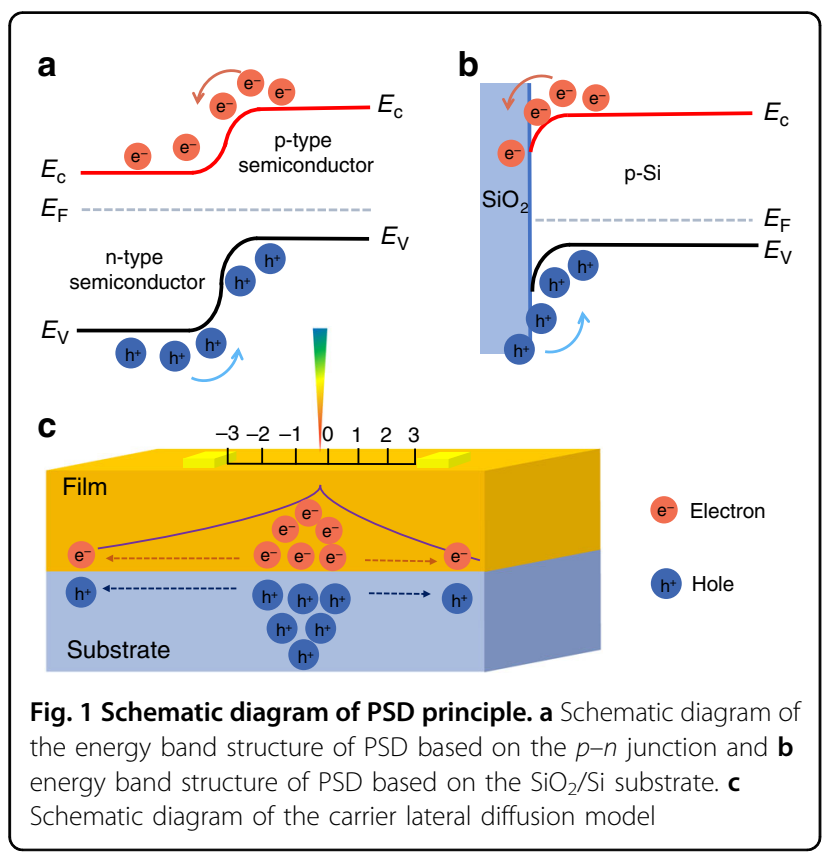

where coefficient $K=\frac{k_{\mathrm{B}} T}{\mathrm{e} \mathrm{n}_{\mathrm{T}}}$, and $L$ and $-L$ are the positions of the two electrodes. Therefore, the voltage difference detected in the two terminal electrodes has a good linear relationship with the laser spot position, making it suitable for PSDs that can detect very small displacements. Silicon-based $p-n$ or $p-\mathrm{i}-n$ junctions are the most commonly used structures for current PSDs, and the response speed was increased from milliseconds in amorphous silicon to microseconds in crystalline silicon devices ${ }^{7}$. PSDs with various architectures have been reported in recent decades, including metalsemiconductor junctions ${ }^{8-13}$, hydrogenated amorphous silicon structures ${ }^{14}$, perovskite materials ${ }^{15-21}$, and twodimensional (2D) materials ${ }^{22-24}$.

The energy band structure and carrier mobility of materials are important factors for the lateral diffusion mechanism of photogenerated carriers. A 2D material is defined as atomically thin layered crystalline sheets with van der Waals interactions between the layers. Graphene and several groups of transition-metal chalcogenides are attractive materials because of their high carrier mobility, tunable energy bandgap, and large light absorptivity ${ }^{25-30}$; these materials have been prepared and successfully developed as field-effect transistors ${ }^{31,32}$, photocatalysts $^{33,34}$, gas sensors ${ }^{35}$, and photodetectors ${ }^{36-38}$ over the past decade. Until now, numerous studies have shown that $2 \mathrm{D}$ materials have great application potential in PSDs $^{25,26,39-42}$. The PSDs based on 2D materials exhibit outstanding properties in a broadband wavelength range with a position sensitivity of up to $401 \mathrm{mV} \mathrm{mm}^{-1} 43$, ultrafast response time of less than $0.5 \mu \mathrm{s}^{43}$, nonlinearity of no more than $\sim 2 \%{ }^{44}$, and a power detection limit as low as $\sim 17 \mathrm{nW}^{6}$. Table 1 shows the performance parameters of various PSDs; obviously, the 2D material-based PSDs satisfy the urgent requirement for highly sensitive, ultrafast photo detection, and low laser power.

Herein, a systematic overview of recent studies on 2D material-based PSDs is provided. The applications of graphene and transition-metal sulfides in PSDs are introduced. The physical mechanism of the large position sensitivity and ultrafast response is elucidated, facilitating new PSD designs.

\section{Graphene-based PSDs}

Graphene consists of a single layer of $\mathrm{sp}^{2}$-hybridized carbon atoms with excellent mechanical, thermal, electrical properties, high chemical stability, and high carrier mobility. Graphene-based devices typically exhibit excellent performance, such as a large photoresponsivity, a high detectivity, a great power conversion efficiency, and an ultrafast response speed. Wang et al. reported a graphene-based PSD that can detect ultrafast weak signals because of the high mobility of graphene and long lifetime of photogenerated carriers at the interface of 
Table 1 Performance parameters of Si-based PSDs

\begin{tabular}{|c|c|c|c|c|}
\hline Structure & Laser wavelength $(\mathrm{nm})$ & Laser power $(\mathrm{mW})$ & Position sensitivity $\left(\mathrm{mV} \mathrm{mm}^{-1} \mathrm{~mW}^{-1}\right)$ & Response time ( $\mu \mathrm{s})$ \\
\hline $\mathrm{Cr} / \mathrm{SiO}_{2} / \mathrm{Si}^{12}$ & 635 & 5 & 8.40 & 1 \\
\hline $\mathrm{Co} / \mathrm{Si}^{13}$ & 832 & 5 & 16.40 & 1 \\
\hline $\mathrm{Ti} / \mathrm{TiO}_{2} / \mathrm{Si}^{10}$ & 632 & 3 & 37.67 & 1 \\
\hline $\mathrm{a}-\mathrm{Si}: \mathrm{H} / \mathrm{c}-\mathrm{Si}{ }^{14}$ & 980 & 8.3 & 0.88 & 1 \\
\hline Graphene/ $/ \mathrm{SiO}_{2} / \mathrm{Si}^{25}$ & 514 & $5 \times 10^{-5}$ & 1 & 1.2 \\
\hline Graphene/Si $i^{6}$ & 532 & $82 \times 10^{-5}$ & 365.85 & 0.44 \\
\hline $\mathrm{a}-\mathrm{MoS}_{2} / \mathrm{Si}^{26}$ & 780 & 10 & 18.3 & 2.1 \\
\hline
\end{tabular}

a

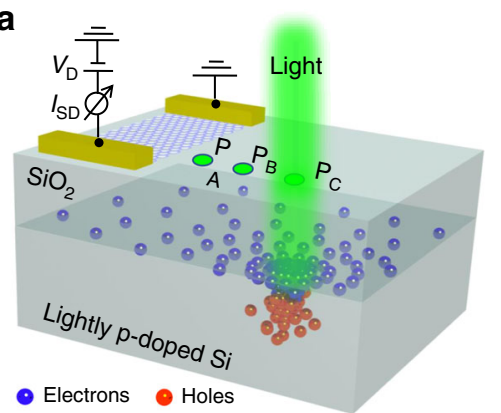

d

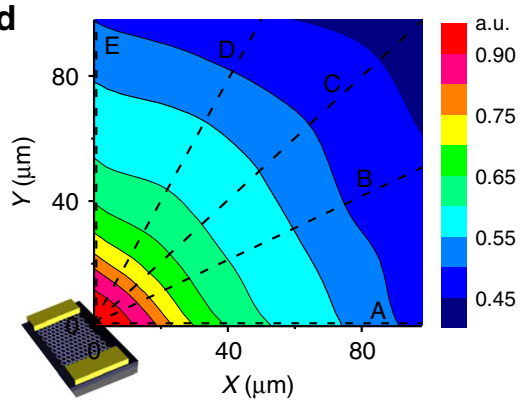

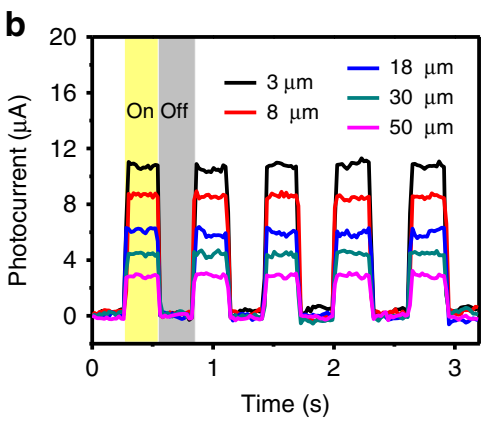

e

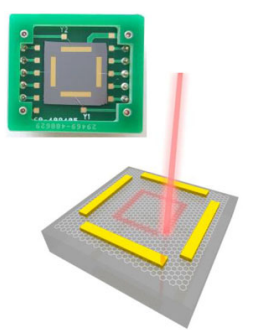

C

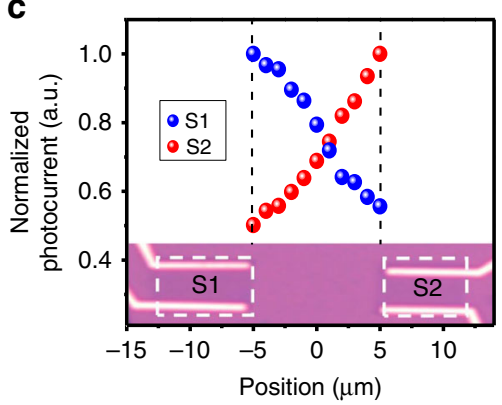

f

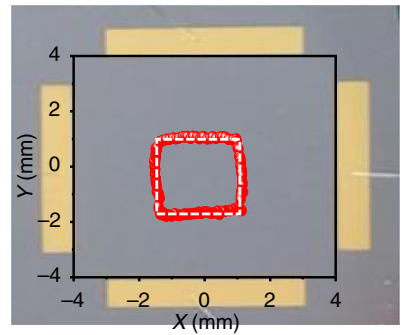

Fig. 2 Graphene-based PSD. a Schematic diagram of graphene-based PSD. b Photoswitching characteristics of graphene-based PSD at different light illumination positions. c Position dependence of the photoresponse of a one-dimensional PSD prepared under 50nW incident light. d 2D spatial mapping of the photocurrent of PSD under 400nW incident light. e Optical image of a 2D PSD and schematic diagram of the movement of light in the operating area. $\mathbf{f}$ Measured trajectory (red dots) of the laser. The white dotted line represents the actual position ${ }^{6,25}$

$\mathrm{SiO}_{2} / \mathrm{Si}^{25}$. Figure $2 \mathrm{a}$ shows a lightly p-doped $\mathrm{Si}$ substrate with a $\mathrm{SiO}_{2}$ layer, and two-terminal graphene is deposited on the top. When light is incident on the surface of the detector, the photogenerated electron-hole pairs are separated by the built-in electric field at the interface of $\mathrm{SiO}_{2}$ and $\mathrm{Si}$ and then spread laterally in the depletion layer. The positional information can be obtained by the correspondence between photoelectric signals collected by the electrodes on graphene and the position of light irradiation. The electrons occupying the acceptor level decrease and the holes occupying the donor level increase due to a large positively charged state from the lightly $p$ doped Si band gap existing at the oxide-silicon interface, making the energy band at the interface bend down. The electrons in the Si conduction band gather at the interface with a lower potential to form a negative depletion layer near the silicon surface; thus, an intrinsic built-in field that can separate the photogenerated carriers is formed.

Thus, the electrons will migrate to the $\mathrm{SiO}_{2} / \mathrm{Si}$ interface and then diffuse laterally until equilibrium is reached, and the magnitude of the photocurrent decreases exponentially with increasing distance from the laser spot. Electrons diffusing into the underlying region of graphene change the hole concentration and channel current by capacitive coupling. This gate effect results in an ultrahigh interfacial amplification $(G)$, determined by the ratio of 
the lifetime $\left(\tau_{1}\right)$ of the photogenerated carrier at the $\mathrm{SiO}_{2} /$ $\mathrm{Si}$ interface and the transit time $\left(\tau_{1}\right)$ in the graphene channel between the two electrodes, and $G=\tau_{1} / \tau_{\mathrm{t}}$. Ultrahigh mobility graphene (approximately $8000 \mathrm{~cm}^{2}$ $\left.\mathrm{V}^{-1} \mathrm{~S}^{-1}\right)$ and the long carrier lifetime of $\mathrm{SiO}_{2} / \mathrm{Si}\left(10^{-6} \mathrm{~s}\right)$ in the graphene-based PSD result in an extremely high G (approximately $10^{4}$ ), thus significantly improving the sensitivity of the device. This interfacial amplification process behaves similarly to a built-in amplifier, which does not increase the signal noise in the graphene channel, so it can significantly improve the sensitivity of the device.

Figure $2 \mathrm{~b}$ shows the photoswitching characteristics of graphene-based PSD at different light illumination positions and the photocurrent signals collected by the electrodes on the graphene. When the laser spot is far away from the graphene, the photocurrent is close to zero because of the isotropic diffusion of carriers, whereas the photocurrent increases when the laser position is close to the graphene channel.

Figure 2c shows the photoresponse characteristics of the PSD under $50 \mathrm{nW}$ laser irradiation with a wavelength of $514 \mathrm{~nm}$. The inset shows the optical image of two devices (S1 and S2) on the same substrate separated by $10 \mu \mathrm{m}$. For device S1, as the laser position increases from -5 to $5 \mu \mathrm{m}$ from the graphene channel, the normalized photocurrent $\left(I_{2}-I_{1}\right) /\left(I_{2}+I_{1}\right)$ decreases from $\sim 1$ to 0.5 , while $\mathrm{S} 2$ increases from 0.5 to 1 . Obviously, the photocurrent of graphene-based PSD performs well. Furthermore, it can also detect the positional changes in $2 \mathrm{D}$ space. Figure $2 \mathrm{~d}$ shows the distribution of photocurrent at different locations. Because the detection limit of photocurrent is $\sim 0.5 \mu \mathrm{A}$, the spatial position resolution of PSD should be less than $1 \mu \mathrm{m}$.

Figure 2e, f show optical images of another graphenebased 2D PSD with a different structure ${ }^{6}$. In this work, Wang et al. designed a high-performance passive PSD based on a graphene-Si hybrid structure. These authors transferred a large area of monolayer graphene to the surface of a lightly $n$-doped Si substrate and produced a prototype PSD with a size of $8 \times 8 \mathrm{~mm}$. Different from the PSD in Fig. 2a, the pinning effect will bend the energy band of the $n$-doped Si surface upward so that the photogenerated holes will enter the graphene layer under the action of the built-in field. A 635-nm laser was used to illuminate the device along a square track (red dots), and the signals collected by the two pairs of electrodes can determine the position of the laser spot in real time (white dots). The red and white dashed squares match; subsequent calculation processing and converting the electrode into a pillow shape can effectively reduce the measurement error. Moreover, the presence of a built-in field in this PSD can effectively separate photogenerated carriers without the need for an external voltage, i.e., there is no extra power consumption during the measurement, making it suitable for portable and integrated devices.
Liu et al. further showed a large-area graphene-Ge Schottky heterojunction ${ }^{45}$. Large-area monolayer graphene $\left(5 \times 5 \mathrm{~mm}^{2}\right)$ is deposited on the $n$-type Ge substrate, as shown in Fig. 3a. The pinning effect originating from the surface state causes the energy band of the depletion layer to bend upward, forming a built-in field with the direction from the Ge substrate to the surface and providing a greater signal collection efficiency for the PSDs. Graphene itself can also absorb light and produce photogenerated carriers, extending the operating wavelength to the near-infrared region (up to $1600 \mathrm{~nm}$ ). The highest position sensitivity of this PSD under $1550 \mathrm{~nm}$ laser irradiation is approximately $50 \mathrm{mV} / \mathrm{mm}$, and the voltage noise is approximately $4 \mu \mathrm{V}$, which is almost negligible. In addition to detecting positional changes, PSDs have many novel features in various applications ${ }^{45}$. As shown in Fig. 3b, angular changes can also be accurately measured with the clever modification of the PSD. When a laser beam is illuminated on the object, the reflected light can be detected by the PSD. If the object rotates at a small angle $\Delta \theta$, then the corresponding reflected light will move $\Delta L$ on the PSD, satisfying the relationship: $\Delta \theta=\frac{1}{2}\left[\arctan \left(\frac{L+\Delta L}{H}\right)-\arctan \left(\frac{L}{H}\right)\right]$. By measuring the displacement $\Delta L$ on the PSD, the angular difference can be obtained. Figure $3 \mathrm{c}$ shows that $\Delta \theta$ is linearly related to $\Delta L$ in the range from -0.05 to 0.05 degrees. According to the high spatial resolution $(0.1 \mu \mathrm{m})$ of graphene-based PSD, the smallest detectable angular difference can be accurate to $5 \times 10^{-6}$ degrees within a working distance of $50 \mathrm{~cm}$.

The fast response characteristics of the PSD can also be used to precisely distinguish the frequency of sound, as shown in Fig. 3d. In this noncontact optical sensing system, the PSD can precisely measure and recover the sound frequency by the following process: The laser beam (I) is irradiated on the speaker surface (II), and the vibration of the speaker causes a small displacement of the reflected light. This displacement can be detected by the PSD (III) and recorded by an oscilloscope (IV). The recorded electrical signal can be converted into sound again through an amplifier (V) and speaker (VI). Figure 3e shows the experimental results of the PSD-based sound recording and storage system. The trajectory tracking of high-speed moving objects has detector requirements of high accuracy and response speed. Figure $3 \mathrm{f}$ shows that the graphene-based PSD can track the trajectory of a target moving at a speed higher than $100 \mathrm{~km} / \mathrm{h}$. The positions of the object (blue dots) can be clearly recorded. Without the limitations of the source meter and software system, the minimum capture interval can be less than $110 \mu \mathrm{s}$. This trajectory tracking has a high spatial resolution and precise trajectory reproduction because the mechanical dither (red dots) can be recorded in real time. 


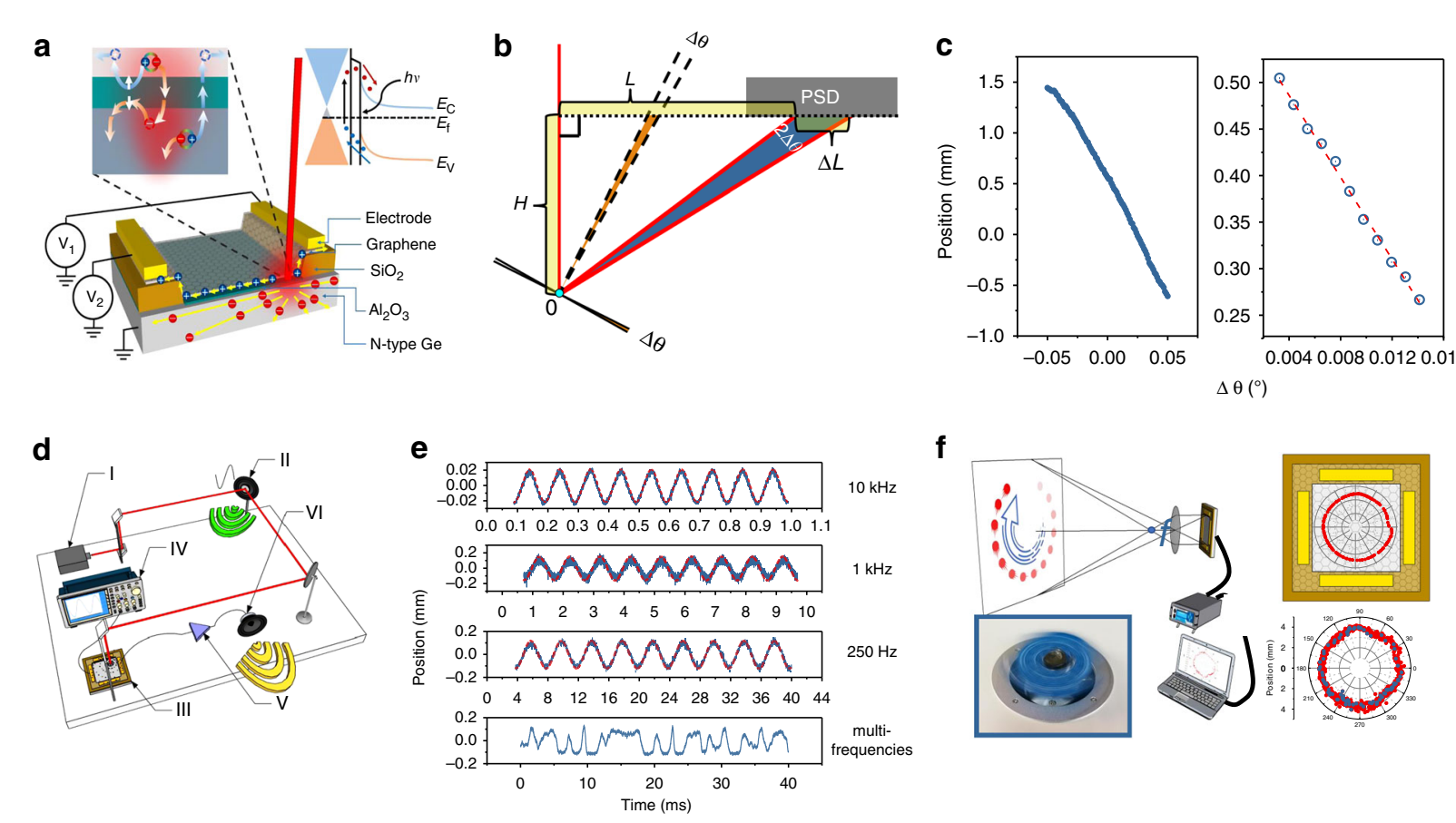

Fig. 3 Multiple applications of graphene-based PSDs. a Schematic diagram of the graphene-Ge PSD. $\mathbf{b}$ Schematic structure of triangulation angle measurement by the PSD. $\mathbf{c}$ Results of the small-angle measurement. $\mathbf{d}$ Setup of the vibration frequency measurement. e Different vibrations recorded by the PSD. $\mathbf{f}$ High-speed trajectory tracking using the graphene-Ge PSD ${ }^{45}$

Therefore, a graphene-based PSD has excellent characteristics, such as a fast response time, high sensitivity, good linearity, and no power consumption, making it a promising candidate for various applications in precise measurements and noncontact optical sensing.

\section{MoS $_{2}$-based PSDs}

As a typical transition-metal dichalcogenide, $\mathrm{MoS}_{2}$ has become one of the most studied 2D materials in recent years. $\mathrm{MoS}_{2}$ has a suitable band gap and a high carrier and light absorbance for optoelectronic devices; however, there are still many problems in preparing large-area and defect-free $\mathrm{MoS}_{2}$, making it difficult to fully exert its brilliant optoelectronic properties in devices. To overcome these drawbacks, the preparation of multilayer $\mathrm{MoS}_{2}$ has been attempted, and a feasible method has been developed for constructing high-performance optoelectronic devices by changing the morphology of $\mathrm{MoS}_{2}$ or by combining it with different semiconductors, such as making $\mathrm{MoS}_{2} / \mathrm{Si}$ and $\mathrm{MoS}_{2} / \mathrm{GaAs}$ junctions ${ }^{23,40}$.

Qiao et al. prepared vertically oriented few-layered $\mathrm{MoS}_{2}$ nanosheets $\left(\mathrm{V}-\mathrm{MoS}_{2}\right)$ using chemical vapor deposition $(\mathrm{CVD})^{43,44}$. These researchers successfully transferred $\mathrm{MoS}_{2}$ nanosheets to a Si substrate to form a self-powered, high-performance PSD. Figure 4a shows a schematic illustration of the PSD based on a V-MoS $/ 2 \mathrm{Si}$ heterojunction. $\mathrm{Ti} / \mathrm{Au}$ electrodes were deposited on the surface of $\mathrm{MoS}_{2}$ nanoplates, and Ag electrodes were plated on the back of the substrate to ensure good signal collection. The band gaps of the $\mathrm{p}-\mathrm{Si}$ substrate $\left(E_{\mathrm{g} 1}\right)$ and $\mathrm{n}-\mathrm{MoS}_{2}\left(E_{\mathrm{g} 2}\right)$ are $1.12 \mathrm{eV}$ and $1.30 \mathrm{eV}$, respectively, as shown in Fig. 4b. When a laser beam is incident on the surface of the heterojunction, the film and substrate absorb the energy of the laser to excite electron-hole pairs. Under the action of the built-in field at the interface, the electrons are swept and tunneled to the $\mathrm{MoS}_{2}$ layer, and the corresponding holes enter the $\mathrm{Si}$ substrate.

Figure 4c shows the typical laser position dependence of the LPE curves of the $\mathrm{MoS}_{2}$-based PSD. When the laser beam moved from one electrode to the other, a linear relationship was observed between the laser position and LPE. By adjusting the laser power of different wavelengths to $5 \mathrm{~mW}$ during the measurement, it was observed that all the curves maintain good linearity. However, the position sensitivity will first increase with increasing wavelength from 405 to $808 \mathrm{~nm}$ and then decrease at a longer wavelength laser irradiation, as shown in the inset of Fig. 4c, indicating that the relationship between the LPE and laser wavelength is nonmonotonic. In addition, the laser power also significantly affects the LPE. Figure $4 \mathrm{~d}$ shows the LPE curves with different laser powers; the inset shows the relationship between laser power and position sensitivity. When the laser power was increased from 0.1 to $80 \mathrm{~mW}$, the 
a
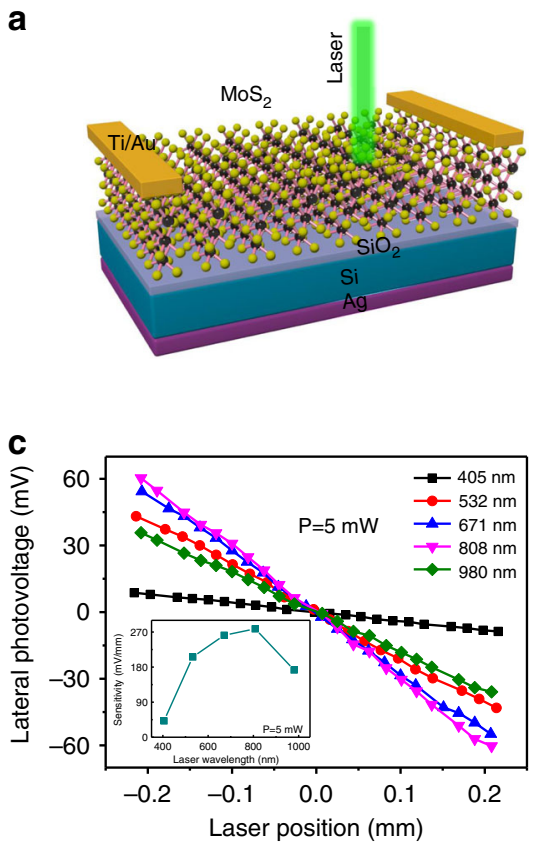

b
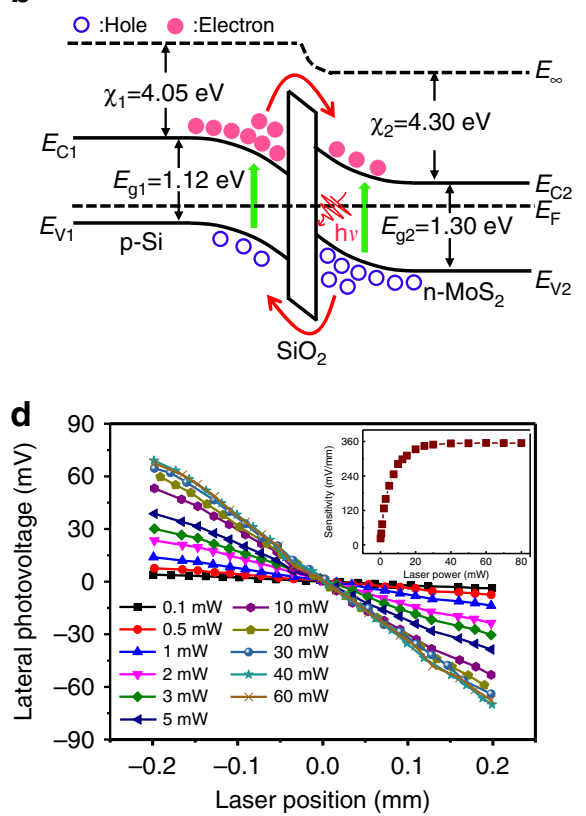

Fig. $4 \mathrm{MoS}_{2}$-based PSD. a Schematic illustration of the lateral photoelectric effect in the $\mathrm{MoS}_{2} / \mathrm{Si}$ heterojunction. $\mathbf{b}$ Energy band diagram of the $\mathrm{MoS}_{2} / \mathrm{Si}$ heterojunction. $\mathbf{c}$ Dependence of the LPE on the laser position for different lasers with an illumination of $5 \mathrm{~mW}$. $\mathbf{d}$ Dependence of the LPE on the laser position with an illumination of a $532 \mathrm{~nm}$ laser at different laser powers ${ }^{43,44}$

position sensitivity first increases exponentially and then becomes saturated. The $\mathrm{MoS}_{2}$ layer has a large bandgap, and its thickness is negligible relative to the $\mathrm{Si}$ wafer. Most of the light absorption occurs in the $\mathrm{Si}$ wafer; therefore, the PSD has the largest response to an $808 \mathrm{~nm}$ wavelength laser. Moreover, the built-in field of heterojunctions has a limited ability to separate the photogenerated carriers. Although an increase in laser power can excite more carriers, it also increases the recombination rate. The number of electron-hole pairs that can be effectively separated is limited. Excess photogenerated carriers recombine quickly, and the LPE cannot be further increased.

Most of the studies believe that high-quality 2D materials are the basis of good LPE performance, but the preparation conditions of a single-crystal $\mathrm{MoS}_{2}$ film are very demanding, significantly limiting its application prospects. Compared with a $\mathrm{MoS}_{2}$ single-crystal film, amorphous $\mathrm{MoS}_{2}\left(\mathrm{a}-\mathrm{MoS}_{2}\right)$ can be easily prepared over a large area on a Si wafer. Hu et al. reported a PSD based on a- $-\mathrm{MoS}_{2} / \mathrm{Si}$ structures with a large LPE and an ultrafast response time ${ }^{26}$. The a- $\mathrm{MoS}_{2}$ films were grown on $p$-type and $n$-type $\mathrm{Si}$ wafers to form $p-n$ and $n-n$ junctions by pulsed laser deposition, respectively, thus achieving two different junctions with opposite-direction built-in fields at the interfaces. The laser excited holes and electrons flow to the a-MoS films in the a-MoS $/ \mathrm{n}-\mathrm{Si}$ and a-MoS $/$ $\mathrm{p}-\mathrm{Si}$ junctions, respectively, making them good candidates to explore the true origin of LPE properties in $\mathrm{Si}$ based PSDs.

Figure 5a shows that the a- $\mathrm{MoS}_{2} / \mathrm{n}-\mathrm{Si}$ and a- $-\mathrm{MoS}_{2} / \mathrm{p}-\mathrm{Si}$ junctions have opposite LPEs, and the LPEs between the electrodes of the two junctions have a good linear dependence on the position of the laser irradiation point. The largest positional sensitivity observed in the a- $\mathrm{MoS}_{2} /$ $\mathrm{n}-\mathrm{Si}$ and a- $\mathrm{MoS}_{2} / \mathrm{p}-\mathrm{Si}$ junctions is 183 and $145 \mathrm{mV} \mathrm{mm}^{-1}$, respectively.

When the a-MoS $\mathrm{M}_{2}$ film is deposited on Si substrates, different band structures of a- $\mathrm{MoS}_{2} / \mathrm{n}-\mathrm{Si}$ and a- $\mathrm{MoS}_{2} / \mathrm{p}-\mathrm{Si}$ junctions are formed due to different Fermi levels of $n-S i$ and $\mathrm{p}$-Si, resulting in different orientations of the built-in field, as shown in the inset of Fig. 5b, c, respectively. The excited electrons and holes flow to the film side and then spread laterally in a-MoS $2 / \mathrm{p}-\mathrm{Si}$ and a- $\mathrm{MoS}_{2} / \mathrm{n}-\mathrm{Si}$ junctions, respectively, providing different effects on the response time. Figure $5 \mathrm{~b}$, $\mathrm{c}$ show a variation in the

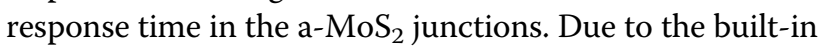
field, minority carriers in the semiconductor will gather at the interface of the heterojunction to form an inversion layer. The thickness of the inversion layer at the $\mathrm{SiO}_{2} / \mathrm{Si}$ interface is less than $5 \mathrm{~nm}$, and the room temperature mobilities of electrons and holes in the inversion layer are approximately 700 and $400 \mathrm{~cm}^{2} \mathrm{~V}^{-1} \mathrm{~s}^{-1}$, respectively. The mobility of electrons in a-MoS $\mathrm{M}_{2}$ films deposited on p-Si substrates is approximately $415 \mathrm{~cm}^{2} \mathrm{~V}^{-1} \mathrm{~s}^{-1}$, and the mobility of holes in a-MoS $\mathrm{M}_{2}$ films deposited on $\mathrm{n}-\mathrm{Si}$ 

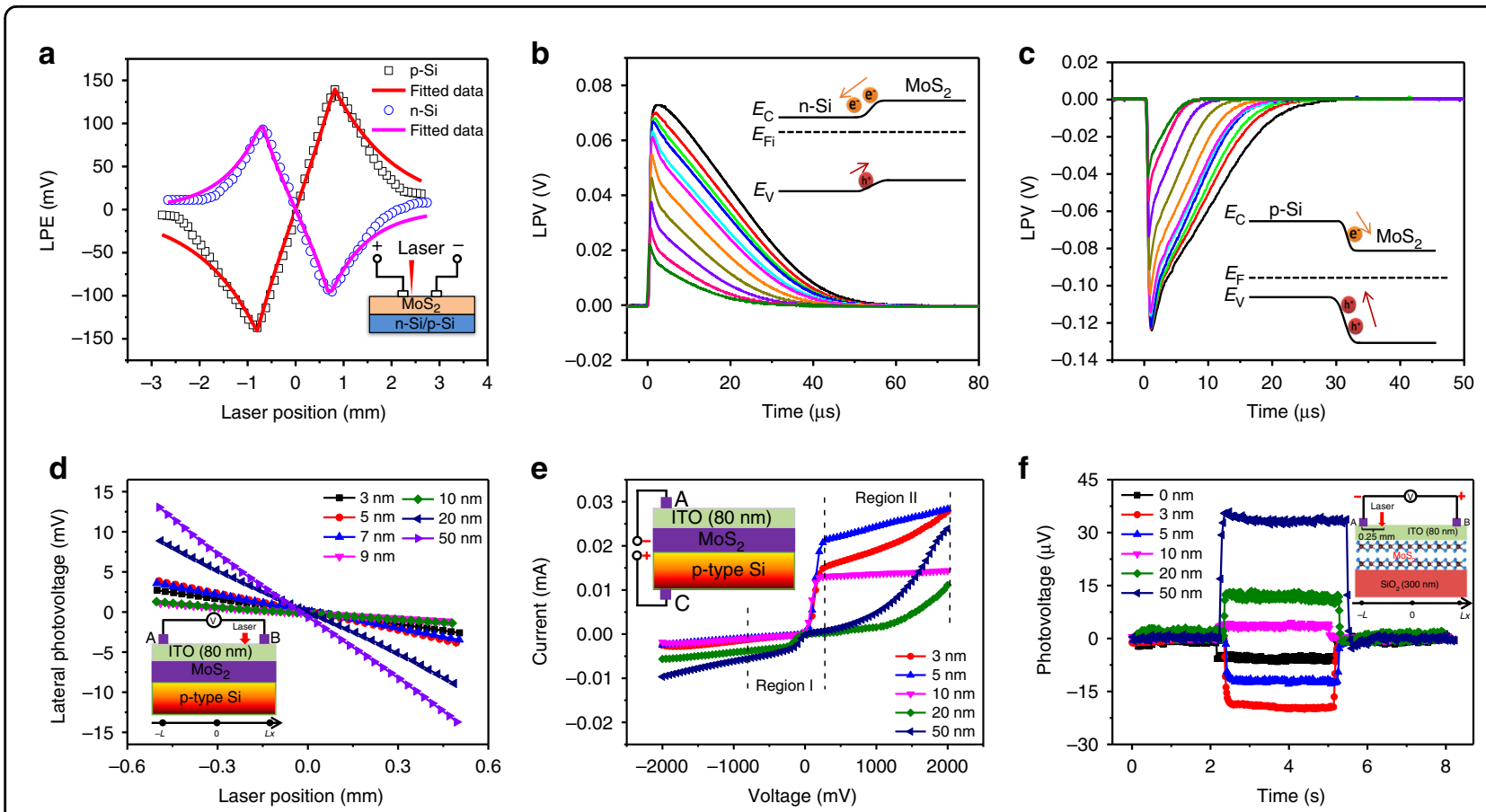

Fig. 5 Large positional sensitivity with fast response time of $\mathbf{M o S}_{2}$-based PSD. a Dependence of the LPE on the illumination position relative to the location of electrodes in a-MoS $/ \mathrm{n}$-Si (blue) and a-MoS $/ \mathrm{p}$-Si (black) junctions. Variation in the LPE with time in $\mathbf{b}$ a-MoS $/ \mathrm{n}-\mathrm{Si}$ and $\mathbf{c}$ a-MoS $/ \mathrm{p}-\mathrm{Si}$ junctions. The insets show schematic diagrams of the energy band structure. $\mathbf{d}$ Dependence of LPE on the laser position for ITO/MoS $/ \mathrm{p}-\mathrm{Si}$ heterojunctions with different $\mathrm{MoS}_{2}$ thicknesses. e Longitudinal I-V curves of ITO/MoS $(3,5,10,20$, and $50 \mathrm{~nm}) / \mathrm{p}-\mathrm{Si}$ heterojunctions. f Timedependent photovoltages in $\mathrm{ITO}_{\mathrm{MoS}}(3,5,10,20$, and $50 \mathrm{~nm}) / \mathrm{SiO}_{2}$ heterojunctions with illumination of a $532 \mathrm{~nm}$ laser, 5,26

substrates is approximately $154 \mathrm{~cm}^{2} \mathrm{~V}^{-1} \mathrm{~s}^{-1}$. These results indicate that most of the photogenerated carriers diffuse laterally in the inversion layer with higher mobilities than in the a-MoS 2 film. When the a-MoS $2 / n-S i$ junction is connected with a $300 \Omega$ resistor, the rise time is $\sim 200 \mathrm{~ns}$, and the relaxation time is reduced to approximately $5.8 \mu \mathrm{s}$. In the a- $\mathrm{MoS}_{2} / \mathrm{p}$-Si junction, the fastest rise time is $\sim 150 \mathrm{~ns}$, and the relaxation time is $\sim 2.1 \mu \mathrm{s}$, nearly three times faster than that in the a-MoS 2 $\mathrm{n}$-Si junction, which is consistent with the different mobilities of electrons and holes in the inversion layer at the interface between $\mathrm{MoS}_{2}$ and Si. These results clearly indicate that the interface of the a- $\mathrm{MoS}_{2} / \mathrm{Si}$ junction is responsible for the LPE.

Qiao et al. found that $\mathrm{MoS}_{2}$ undergoes n-type to p-type transformation with increasing thickness in the ITO/ $\mathrm{MoS}_{2} / \mathrm{p}$-Si heterojunction, and the LPE significantly improved in a wide spectrum response ranging from visible to near-infrared ${ }^{5}$. Figure $5 \mathrm{~d}$ shows the LPE of ITO/ $\mathrm{MoS}_{2} / \mathrm{p}$-Si heterostructures under illumination with a 532-nm laser. When the thickness of $\mathrm{MoS}_{2}$ is less than $9 \mathrm{~nm}$, the LPE first increases and then decreases with an increase in the film thickness. As the film thickness continues to increase, the LPE increases again, indicating that the thickness of $\mathrm{MoS}_{2}$ plays an important role in the transport of photogenerated carriers. To further elucidate the mechanism, the longitudinal $I-V$ curves of the heterojunction were measured, as shown in Fig. 5e. With an increase in the thickness of $\mathrm{MoS}_{2}$, the Schottky barrier in region I (representing the characteristic between $\mathrm{p}-\mathrm{Si}$ and $\mathrm{MoS}_{2}$ ) is enhanced, but in region II (representing the characteristic between the ITO layer and $\mathrm{MoS}_{2}$ ), it is reduced. When the thickness of $\mathrm{MoS}_{2}$ increases from 5 to $10 \mathrm{~nm}$, the Schottky barrier in both regions I and II is reduced, and the carriers could hardly be transmitted from the $\mathrm{MoS}_{2}$ layer to the ITO layer, so the current is greatly decreased. As the thickness of $\mathrm{MoS}_{2}$ increases again, the Schottky barrier in region I still decreases. However, the Schottky barrier in region II is significantly improved, and the built-in field can accelerate photogenerated electron transfer from $\mathrm{p}-\mathrm{Si}$ to the ITO layer, causing the LPE to rapidly increase again.

The photovoltaic response of ITO/MoS $/ \mathrm{SiO}_{2}$ samples with different $\mathrm{MoS}_{2}$ thicknesses was measured, as shown in Fig. 5f. Two electrodes with a pitch of $1.0 \mathrm{~mm}$ were plated on the surface of each sample, and a laser $(532 \mathrm{~nm}$, $10 \mathrm{~mW}$ ) was used to irradiate at a point with a distance of $0.25 \mathrm{~mm}$ from the electrode. It was observed that when the thickness of $\mathrm{MoS}_{2}$ is less than $10 \mathrm{~nm}$, the photovoltage is always negative, while the photovoltage of samples with thicker films changed from negative to positive and increased rapidly with increasing $\mathrm{MoS}_{2}$ thickness. These 
results indicate that the built-in field at the interface is reversed due to the n-type to $\mathrm{p}$-type transformation of $\mathrm{MoS}_{2}$.

The built-in field at the interface of 2D materials and semiconductor substrates plays an important role in the performance of PSDs. The transmission direction of photogenerated carriers and response time can be effectively tuned by changing the band structure or thickness of the film, which is meaningful for the design of new PSDs.

\section{Conclusions and outlook}

The rise of 2D materials has aroused great interest in new PSDs. To achieve higher-performance PSDs, various strategies have been used with different 2D materials. By studying the low-energy consumption, high sensitivity, and ultrafast response sensing systems based on 2D materials such as graphene and transition sulfides, the physical mechanisms of the large LPE and ultrafast response time in the PSD were elucidated. We believe that the high mobility and absorption of $2 \mathrm{D}$ materials are important factors to achieve a high-performance PSD. The high mobility provides a guarantee for the high-speed transmission of a large number of photogenerated carriers, which is important for the response speed of the PSD. High absorption can effectively increase the number of photogenerated carriers and enhance the sensitivity of the PSD. In addition, a PSD that combines 2D materials with various semiconductors not only effectively utilizes the high carrier mobility and light absorption of $2 \mathrm{D}$ materials but also uses the built-in field to spontaneously separate photogenerated carriers. We believe that the effective use of the characteristics of $2 \mathrm{D}$ materials to prepare high-sensitivity, fast-response, and other highperformance PSDs is the main research direction in the future. Combining the advantages of different 2D materials in one detector, such as using the high mobility of graphene and the suitable band structure of $\mathrm{MoS}_{2}$ to produce high-performance and low-consumption detectors, is also of great significance. Furthermore, there are still many challenges and opportunities for PSDs based on $2 \mathrm{D}$ materials in future research. For example, reducing the technical difficulty of large-area film preparation is a prerequisite for the development of multipoint and image detection functions. Because of the limitations of the energy band of Si and 2D materials, current PSDs based on 2D materials mainly absorb visible and near-infrared light, and the development of UV PSDs can provide a wider application range.

\section{Acknowledgements}

This work is supported by the National Natural Science Foundation of China (Grant Nos. 51672057, 51722205, 51772067, and 51902091), the International Science \& Technology Cooperation Program of China (2012DFR50020), the Fundamental Research Funds for the Central Universities (Grant No. HIT.
BRETIV.201801), the Natural Science Foundation of Heilongjiang Province (Grant No. E2018032), and the Program for New Century Excellent Talents in University (NCET-13-0174).

Conflict of interest

The authors declare that they have no conflict of interest.

Received: 11 January 2020 Revised: 18 March 2020 Accepted: 31 March 2020

Published online: 20 May 2020

\section{References}

1. Henry, J. \& Livingstone, J. Thin-film amorphous silicon position-sensitive detectors. Adv. Mater. 13, 1023-1026 (2001).

2. Boeringer, D. W. \& Tsu, R. Lateral photovoltaic effect in porous silicon. Appl. Phys. Lett. 65, 2332-2334 (1994).

3. Jin, K. J. et al. Dember effect induced photovoltage in perovskite $p-n$ heterojunctions. Appl. Phys. Lett. 91, 081906 (2007).

4. Zhang, B., Du, L. \& Wang, H. Bias-assisted improved lateral photovoltaic effect observed in $\mathrm{Cu}_{2} \mathrm{O}$ nano-films. Opt. Express 22, 1661-1666 (2014).

5. Qiao, S. et al. Large lateral photovoltage observed in $\mathrm{MoS}_{2}$ thicknessmodulated ITO/MoS $/$ p-Si heterojunctions. ACS Appl. Mater. Interfaces 9, 18377-18387 (2017).

6. Wang, W. H. et al. High-performance position-sensitive detector based on graphene-silicon heterojunction. Optica 5, 27-31 (2018).

7. Vieira, M. Speed photodetectors based on amorphous and microcrystalline silicon p-i-n devices. Appl. Phys. Lett. 70, 220-222 (1997).

8. Levine, B. F. et al. Lateral photoeffect in thin amorphous superlattice films of Si and Ti grown on a Si substrate. Appl. Phys. Lett. 49, 1537-1539 (1986).

9. Xiao, S. Q. et al. The Co-film-thickness dependent lateral photoeffect in $\mathrm{Co}-\mathrm{SiO}_{2}-\mathrm{Si}$ metal-oxide-semiconductor structures. Opt. Express 16, 3798-3806 (2008).

10. Yu, C. Q., Wang, H. \& Xia, Y. X. Giant lateral photovoltaic effect obsenved in $\mathrm{TiO}_{2}$ dusted metal-semiconductor structure of $\mathrm{Ti}_{\mathrm{TiO}} / \mathrm{Si}$. Appl. Phys. Lett. 95, 141112 (2009).

11. Liu, S. et al. Lateral photovoltaic effect observed in nano Au film covered twodimensional colloidal crystals. Appl. Phys. Lett. 104, 111110 (2014).

12. Liu, S., Xie, X. \& Wang, H. Lateral photovoltaic effect and electron transport observed in Cr nano-film. Opt. Express 22, 11627-11632 (2014).

13. Yu, C. Q. \& Wang, H. Large near-infrared lateral photovoltaic effect observed in Co/Si metal-semiconductor structures. Appl. Phys. Lett. 96, 171102 (2010).

14. Qiao, S. et al. Large lateral photovoltaic effect in a-Si: $\mathrm{H} / \mathrm{c}-\mathrm{Si} \mathrm{p}-\mathrm{i}-\mathrm{n}$ structure with the aid of bias voltage. Appl. Phys. Express 8, 122201 (2015).

15. Lu, H. B. et al. Picosecond photoelectric characteristic in $\mathrm{La}_{0.7} \mathrm{Sr}_{0.3} \mathrm{MnO}_{3} / \mathrm{Si} p-n$ junctions. Appl. Phys. Lett. 86, 241915 (2005).

16. Zhao, K. et al. Transient lateral photovoltaic effect in $p$ - $n$ heterojunctions of $\mathrm{La}_{0.7} \mathrm{Sr}_{0.3} \mathrm{MnO}_{3}$ and Si. Appl. Phys. Lett. 88, 141914 (2006).

17. Wang, X. J. et al. Self-powered ultraviolet vertical and lateral photovoltaic effect with fast-relaxation time in $\mathrm{NdNiO}_{3} / \mathrm{Nb}$ : $\mathrm{SrTiO}_{3}$ heterojunctions. Appl. Phys. Lett. 112, 122103 (2018)

18. Gu, L. L. \& Fan, Z. Y. Perovskite/organic-semiconductor heterojunctions for ultrasensitive photodetection. Light Sci. Appl. 6, e17090 (2017)

19. Xie, C. et al. Ultrasensitive broadband phototransistors based on perovskite/ organic-semiconductor vertical heterojunctions. Light Sci. Appl. 6, e17023 (2017).

20. Hu, W. et al. Germanium/perovskite heterostructure for high-performance and broadband photodetector from visible to infrared telecommunication band. Light Sci. Appl. 8, 106 (2019).

21. Yang, T. F. et al. Ultrahigh-performance optoelectronics demonstrated in ultrathin perovskite-based vertical semiconductor heterostructures. ACS Nano 13, 7996-8003 (2019)

22. Zhang, Q. et al. Solution-processed graphene quantum dot deep-UV photodetectors. ACS Nano 9, 1561-1570 (2015).

23. Xiao, P. et al. Solution-processed 3D RGO-MoS 2 /pyramid Si heterojunction for ultrahigh detectivity and ultra-broadband photodetection. Adv. Mater. 30, 1801729 (2018).

24. Wu, F. et al. High efficiency and fast van der Waals hetero-photodiodes with a unilateral depletion region. Nat. Commun. 10, 4663 (2019). 
25. Wang, W. H. et al. Interfacial amplification for graphene-based positionsensitive-detectors. Light Sci. Appl. 6, e17113 (2017).

26. $\mathrm{Hu}, \mathrm{C}$. et al. Origin of the ultrafast response of the lateral photovoltaic effect in amorphous $\mathrm{MoS}_{2} / \mathrm{Si}$ junctions. ACS Appl. Mater. Interfaces 9, 18362-18368 (2017).

27. Shan, H. Y. et al. Direct observation of ultrafast plasmonic hot electron transfer in the strong coupling regime. Light Sci. Appl. 8, 9 (2019).

28. Zhu, L. X. et al. Angle-selective perfect absorption with two-dimensional materials. Light Sci. Appl. 5, e16052 (2016).

29. Zheng, Z. B. et al. Tailoring of electromagnetic field localizations by twodimensional graphene nanostructures. Light Sci. Appl. 6, e17057 (2017).

30. Zhao, X. N. et al. Reversible alternation between bipolar and unipolar resistive switching in $\mathrm{Ag} / \mathrm{MoS}_{2} / \mathrm{Au}$ structure for multilevel flexible memory. J. Mater. Chem. C 6, 7195-7200 (2018).

31. Radisavljevic, B. et al. Single-layer $\mathrm{MoS}_{2}$ transistors. Nat. Nanotechnol. 6 , 147-150 (2011).

32. Late, D. J. et al. Hysteresis in single-layer $\mathrm{MoS}_{2}$ field effect transistors. ACS Nano 6, 5635-5641 (2012).

33. Xiang, Q. J., Yu, J. G. \& Jaroniec, M. Synergetic effect of $\mathrm{MoS}_{2}$ and graphene as cocatalysts for enhanced photocatalytic $\mathrm{H}_{2}$ production activity of $\mathrm{TiO}_{2}$ nanoparticles. J. Am. Chem. Soc. 134, 6575-6578 (2012).

34. Zhao, X. N. et al. Photocatalytic reduction of graphene oxide- $\mathrm{TiO}_{2}$ nanocomposites for improving resistive-switching memory behaviors. Small 14, 1801325 (2018).

35. He, Q. Y. et al. Fabrication of flexible $\mathrm{MoS}_{2}$ thin-film transistor arrays for practical gas-sensing applications. Small 8, 2994-2999 (2012).
36. Wi, S. et al. Enhancement of photovoltaic response in multilayer $\mathrm{MoS}_{2}$ induced by plasma doping. ACS Nano 8, 5270-5281 (2014).

37. Tsai, M. L. et al. Monolayer $\mathrm{MoS}_{2}$ heterojunction solar cells. ACS Nano 8 8317-8322 (2014)

38. Cakmakyapan, S. et al. Gold-patched graphene nano-stripes for highresponsivity and ultrafast photodetection from the visible to infrared regime. Light Sci. Appl. 7, 20 (2018).

39. Javadi, M., Gholami, M. \& Abdi, Y. IR position-sensitive detectors based on double-junction asymmetric $\mathrm{TiO}_{2} / \mathrm{MoS}_{2} /$ reduced graphene-oxide sandwiches. J. Mater. Chem. C 6, 8444-8452 (2018).

40. Hao, L. Z. et al. Large lateral photovoltaic effect in $\mathrm{MoS}_{2} / \mathrm{GaAs}$ heterojunction. Nanoscale Res. Lett. 12, 562 (2017).

41. Wang, $X$. J. et al. Large lateral photovoltaic effect with ultrafast relaxation time in SnSe/Si junction. Appl. Phys. Lett. 109, 023502 (2016)

42. Zhang, Y. et al. Ultrahigh position sensitivity and fast optical relaxation time of lateral photovoltaic effect in $\mathrm{Sb}_{2} \mathrm{Se}_{3} / p$-Si junctions. Opt. Express 26, 34214-34223 (2018).

43. Cong, R. D. et al. Ultrahigh, ultrafast, and self-powered visible-near-infrared optical position-sensitive detector based on a CVD-prepared vertically standing few-layer $\mathrm{MoS}_{2} /$ Si heterojunction. Adv. Sci. 5, 1700502 (2018).

44. Qiao, S. et al. Laser-induced photoresistance effect in Si-based vertical standing $\mathrm{MoS}_{2}$ nanoplate heterojunctions for self-powered high performance broadband photodetection. J. Mater. Chem. C 7, 10642-10651 (2019).

45. Liu, K. Y. et al. Graphene-based infrared position-sensitive detector for precise measurements and high-speed trajectory tracking. Nano Lett. 19, 8132-8137 (2019). 